\title{
UNDERSTANDING THE CHIRAL GEOMETRY WITH $K$-PLOT AND AZIMUTHAL-PLOT*
}

\author{
F.Q. $\mathrm{CHEN}^{\mathrm{a}}$, J. MenGa,b,c

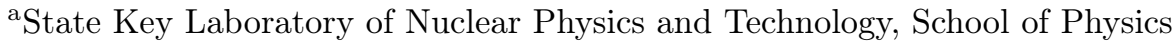 \\ Peking University, Beijing 100871, China \\ ${ }^{b}$ Yukawa Institute for Theoretical Physics, Kyoto University \\ Kyoto 606-8502, Japan \\ ${ }^{\mathrm{c}}$ Department of Physics, University of Stellenbosch, Stellenbosch, South Africa
}

(Received December 28, 2017)

The chiral geometry of the angular momentum in the intrinsic frame is extracted from angular momentum projected wave functions in the laboratory frame by the $K$-Plot and Azimuthal-Plot. The method is demonstrated by an application to the chiral doublet bands in ${ }^{128} \mathrm{Cs}$, based on the standard pairing-plus-quadrupole Hamiltonian. The observed energy spectra and the electromagnetic transitions are well-reproduced, and the $K$-Plot and Azimuthal-Plot obtained give evolution of angular momentum geometry with spin, by which the chirality is demonstrated.

DOI:10.5506/APhysPolBSupp.11.117

\section{Introduction}

Since its first prediction by Frauendorf and Meng in 1997 [1], the spontaneous chiral symmetry breaking in triaxial nuclei has attracted intensive attention. The spontaneous chiral symmetry breaking is due to the aplanar orientation of the angular momentum with respect to the intrinsic frame, in which case the angular momentum has non-vanishing components on all the three principle axes, contributed by the valence hole(s), the valence particle(s), and the rotating triaxial core, respectively. These three components can form a left-handed or a right-handed configuration, by which the spontaneous breaking of chiral symmetry is defined.

The spontaneous symmetry breaking in the intrinsic frame manifests itself in the laboratory frame by the chiral doublet bands, which is a pair of $\Delta I=1$ bands with the same parity. The chiral doublet bands are characterized by the near degenerate energy spectra and specific behaviors in the

* Presented at the XXIV Nuclear Physics Workshop "Marie and Pierre Curie", Kazimierz Dolny, Poland, September 20-24, 2017. 
electromagnetic transitions. The first examples of chiral doublet bands were observed in 2001 in four isotones with $N=75$ in the $A \sim 130$ region [2]. Since then, more than 40 candidates of chiral doublet bands have been observed in mass regions $A \sim 80$ [3,4], $A \sim 100$ [5-10], $A \sim 130$ [2,11-16] and $A \sim 190[17,18]$ (see Refs. [19-21] for details).

The first theoretical prediction for chiral doublet bands is based on the particle rotor model (PRM) and the tilted axis cranking (TAC) approaches, which have become the mainstream theoretical methods adopted in the studies of nuclear chirality. The advantage of the PRM is that it treats the nuclear rotation in a fully quantum mechanical way, with the angular momentum conserved as a good quantum number. Therefore, the PRM generates energy spectra and electromagnetic transitions that can be directly compared with the data. PRM has been generalized to include the pairing correlations [22-26], and many-quasiparticle configurations [27-29]. Satisfactory descriptions for chiral doublet bands have been given for ${ }^{78,80} \mathrm{Br}[3,4]$, ${ }^{103,105,106} \mathrm{Rh}[24,28],{ }^{126} \mathrm{Cs}$ [22], ${ }^{135} \mathrm{Nd}$ [27], and ${ }^{198} \mathrm{Tl}$ [18]. Multiple chiral bands [29-31] are studied as well.

TAC approach described in Ref. [1] is a semi-classical approximation of the PRM. TAC approach can be combined with the Woods-Saxon (or Nilsson) plus Strutinsky shell correction methods, or density functionals. TAC has been used to describe chiral doublet bands in ${ }^{134} \mathrm{Pr}$ and ${ }^{188} \mathrm{Ir}$ [32], and in ${ }^{132} \mathrm{La}[33,34]$. Recently, the three-dimentional TAC based on the covariant density functional theory (CDFT) has been developed and applied for the multiple chirality in ${ }^{106} \mathrm{Rh}$ [35].

As the mean-field approximation gives only the energy minimum, TAC calculations could not provide the energy spectra for the partner band, as well as the characteristic pattern for electromagnetic transitions. In order to describe these results, one has to go beyond mean field by random-phaseapproximation (RPA) or collective Hamiltonian calculations, e.g., TAC+ RPA description for the chiral vibration in the $A \sim 130$ region [36]. However, the RPA does not work for static chirality as it only deals with oscillations with small amplitudes. The large amplitude collective modes of angular momentum in the intrinsic frame can be taken into account by the collective Hamiltonian $[37,38]$. Although, so far, it is based on the simplest version of $\mathrm{TAC}$, it is promising to combine with the microscopic TAC in future.

Although TAC calculations have achieved great success in the studies of chiral doublet bands, they suffer from the broken rotational symmetry, as well as the semi-classical approximation. While the rotational symmetry is conserved in the PRM, it has the disadvantage on the assumption of a core, and the relevant parameters. Therefore, it is natural to seek for new tools for chiral doublet bands. The angular momentum projection approach [39] seems to be a good candidate. It can be based on fully microscopic 
mean field and with the broken rotational symmetry restored by projection. The angular momentum projection approach has been combined with the Nilsson+BCS method [40] and the energy density functionals such as Skyrme [41], Gogny [42,43], as well as the CDFT [44, 45]. It also gives satisfactory descriptions to various excitation phenomenon in deformed nuclei, including the back-bending, the $\beta$ - and $\gamma$-vibrations, the high- $K$ isomers. A recent review can be found in Ref. [46].

As an implementation of the angular momentum projection approach, the projected shell model [40] has been applied to triaxial nuclei with unpaired quasiparticles [47-50], and attempts for understanding the chiral doublet bands have been made $[49,50]$. Although satisfactory description for the data are obtained, it is found challenging to extract the angular momentum geometry in the intrinsic frame.

In the PRM description, the angular momentum geometry can be illustrated in terms of the so-called $K$-distribution, which gives distributions of the components of the angular momentum on the three intrinsic axes obtained immediately from the PRM wave functions. This is not the case for the angular momentum projection, as the projected basis forms a nonorthogonal set. In the TAC calculations, the angular momentum geometry is given explicitly by the tilted angles, which are the polar angle and the azimuthal angle of the angular momentum in the intrinsic frame. The tilted angles have never been discussed with the angular momentum projected descriptions, as the wave functions in this case are defined in the laboratory frame. Therefore, the illustration of the chiral geometry in the angular momentum projection approach remains an open problem.

In this work, we present the chiral geometry within the framework of the angular momentum projection approach reported in Ref. [51]. By K-Plot and Azimuthal-Plot, the angular momentum geometry can be extracted from the angular momentum projected wave functions, by which the chirality is shown explicitly. The typical chiral doublet bands in ${ }^{128} \mathrm{Cs}$ [14] are investigated here as an example.

\section{Sketch of the model}

The starting point is the standard pairing-plus-quadrupole Hamiltonian [39]

$$
\hat{H}=\hat{H}_{0}-\frac{\chi}{2} \sum_{\mu=-2}^{2} \hat{Q}_{\mu}^{+} \hat{Q}_{\mu}-G_{M} \hat{P}^{+} \hat{P}-G_{Q} \sum_{\mu=-2}^{2} \hat{P}_{\mu}^{+} \hat{P}_{\mu},
$$

which includes the spherical single-particle Hamiltonian $H_{0}$, the quadrupolequadrupole interaction, the monopole pairing and the quadrupole pairing. The parameters, including the strengths of the spin-orbit terms and the two-body interactions, are taken from Ref. [47]. 
The intrinsic state $\left|\Phi_{\nu_{0} \pi_{0}}\right\rangle$ for odd-odd nuclei is a two-quasiparticle state on an even-even vacuum $\left|\Phi_{0}\right\rangle$

$$
\left|\Phi_{\nu_{0} \pi_{0}}\right\rangle=\beta_{\nu_{0}}^{+} \beta_{\pi_{0}}^{+}\left|\Phi_{0}\right\rangle,
$$

in which $\nu_{0}$ and $\pi_{0}$ represent orbitals occupied by the unpaired neutron and proton, respectively, and $\beta_{\nu_{0}}^{+}, \beta_{\pi_{0}}^{+}$are the corresponding quasiparticle creation operators. The even-even vacuum $\left|\Phi_{0}\right\rangle$ and its corresponding set of quasiparticle operators $\left\{\beta_{\nu}^{+}, \beta_{\pi}^{+}\right\}$are obtained from the variational calculation

$$
\delta\left\langle\Phi_{\nu_{0} \pi_{0}}\left|\hat{H}-\lambda_{n} \hat{N}-\lambda_{p} \hat{Z}-\lambda_{q_{0}} \hat{Q}_{0}-\lambda_{q_{2}} \hat{Q}_{2}\right| \Phi_{\nu_{0} \pi_{0}}\right\rangle=0 .
$$

In Eq. (3), the Lagrange multipliers $\lambda_{n}$ and $\lambda_{p}$ are determined by the constraint on the particle numbers, while $\lambda_{q_{0}}$ and $\lambda_{q_{2}}$ are determined by the deformation parameters $\beta$ and $\gamma$. For ${ }^{128} \mathrm{Cs}$, the quadrupole deformations constrained are $\beta=0.2$ and $\gamma=30^{\circ}$, which is close to those in Ref. [47] and the results of the CDFT calculation [21]. We block the fourth singleparticle orbital in the $\nu h_{11 / 2}$ subshell, and the lowest single-particle orbital in the $\pi h_{11 / 2}$ subshell. The intrinsic space is spanned by the states $\left|\Phi_{\kappa}\right\rangle \in\left\{\left|\Phi_{\nu_{0} \pi_{0}}\right\rangle,\left|\Phi_{\nu_{0} \bar{\pi}_{0}}\right\rangle,\left|\Phi_{\bar{\nu}_{0} \pi_{0}}\right\rangle,\left|\Phi_{\bar{\nu}_{0} \bar{\pi}_{0}}\right\rangle\right\}$, where $\bar{\nu}_{0}$ and $\bar{\pi}_{0}$ denote the timereversal conjugates of $\nu_{0}$ and $\pi_{0}$.

The projected states

$$
\left\{\hat{P}_{M K}^{I}\left|\Phi_{N, Z, \kappa}\right\rangle\right\} \equiv\left\{\hat{P}_{M K}^{I} \hat{P}^{N} \hat{P}^{Z}\left|\Phi_{\kappa}\right\rangle\right\}
$$

form the basis on which the wave function is expanded

$$
\left|\Psi_{I M}\right\rangle=\sum_{K, \kappa} f_{K, \kappa}^{I} \hat{P}_{M K}^{I}\left|\Phi_{N, Z, \kappa}\right\rangle=\sum_{K, \kappa} f_{K, \kappa}^{I} \frac{2 I+1}{8 \pi^{2}} \int \mathrm{d} \Omega D_{M K}^{I *}(\Omega) \hat{R}(\Omega)\left|\Phi_{N, Z, \kappa}\right\rangle .
$$

The expanding coefficients $f_{K, \kappa}^{I}$ and the eigen-energies $E_{I}$ in Eq. (5) are determined by the Hill-Wheeler equation

$$
\sum_{K^{\prime} \kappa^{\prime}}\left(\left\langle\Phi_{N, Z, \kappa}\left|\hat{H} \hat{P}_{K K^{\prime}}^{I}\right| \Phi_{N, Z, \kappa^{\prime}}\right\rangle-E_{I}\left\langle\Phi_{N, Z, \kappa}\left|\hat{P}_{K K^{\prime}}^{I}\right| \Phi_{N, Z, \kappa^{\prime}}\right\rangle\right) f_{K^{\prime} \kappa^{\prime}}^{I}=0 .
$$

The calculated energy spectra and the electromagnetic transition probabilities for the chiral doublet bands in ${ }^{128} \mathrm{Cs}$ are in a good agreement with the data, the details can be found in Ref. [51].

\section{3. $K$-Plot}

The orientation of the angular momentum can be illustrated by the socalled $K$-Plot, which gives the distributions of the components of the angular 
momentum on the three intrinsic axes. The quantity $K$ in the projected wave function (5) represents the component of the angular momentum on the intrinsic 3 axis [40]. The 3 axis is the long (1-) axis with the triaxial deformation $\gamma$ taken from the sextant $\gamma \in\left[0^{\circ}, 60^{\circ}\right]$, and it could become the short (s-) axis and the intermediate (i-) axis with $\gamma \in\left[120^{\circ}, 180^{\circ}\right]$ and $\gamma \in\left[240^{\circ}, 300^{\circ}\right]$, respectively. Therefore, the distributions of $K_{\mathrm{l}}, K_{\mathrm{s}}$ and $K_{\mathrm{i}}$ can be calculated from the wave function (5) by taking $\gamma$ values from different sextants.

The $K$-distributions are obtained by regarding $\{K, \kappa\}$ in the wave function (5) as generator coordinates. The details can be found in Ref. [51].

The $K$-Plots for spins $I=10,12,14,16,18 \hbar$ are shown in Fig. 1 , in which the evolution of the angular momentum geometry with spin is shown. At $I=10 \hbar$, the peak of the $K_{\mathrm{i}}$-distribution appears at $K_{\mathrm{i}} \sim 1 \hbar$ for band

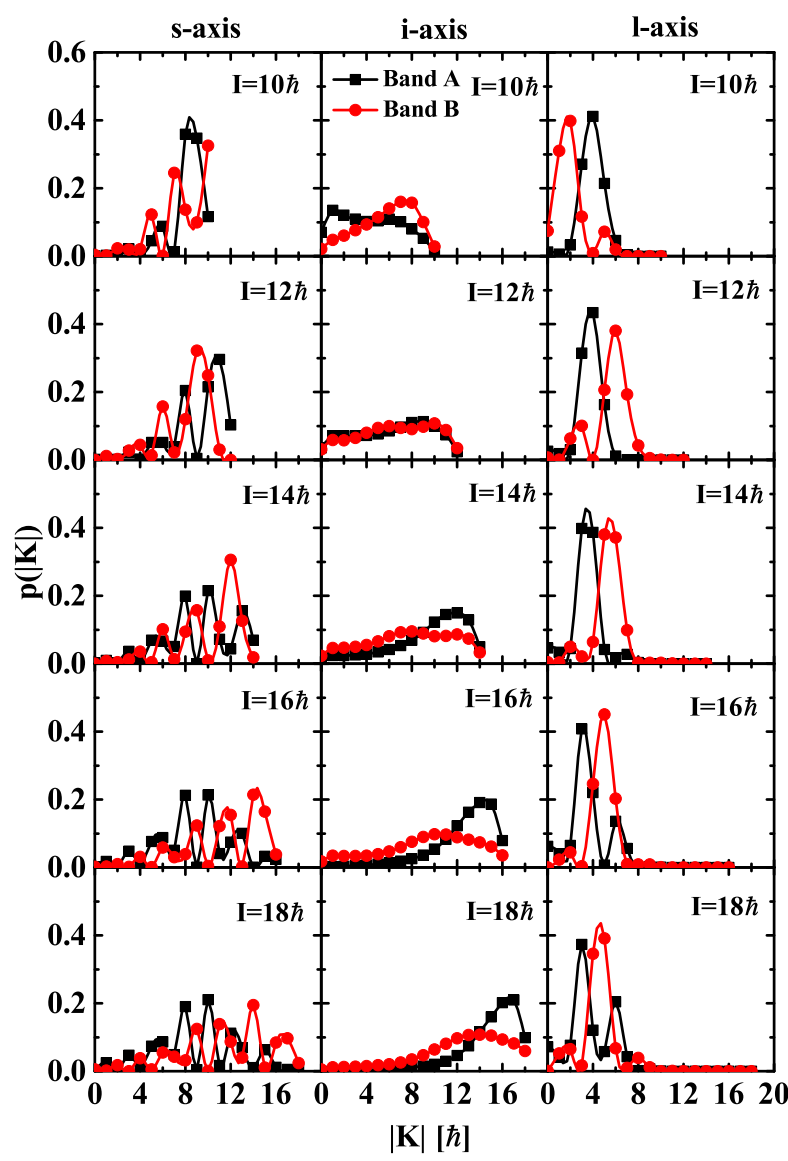

Fig. 1. (Color online) $K$-Plots for the angular momentum on the short (s-), intermediate (i-) and long (l-) axes, calculated for $I=10,12,14,16,18 \hbar$. 
A, which is small compared to the peak values of $K_{\mathrm{s}}$ and $K_{\mathrm{l}}$. This means that for band $\mathrm{A}$, the angular momentum stays within the $\mathrm{s}-1$ plane. For band $\mathrm{B}$, the peak value of $K_{1}$ appears at $K_{1} \sim 2 \hbar$, which is also small compared to those of $K_{\mathrm{s}}$ and $K_{\mathrm{i}}$, suggesting a planar rotation within the s-i plane. Therefore, the $K$-Plots at $I=10 \hbar$ show that both bands stay mainly within a principle plane with possible chiral vibration near the band head.

At $I=12,14 \hbar$, the maximum probability for $K_{\mathrm{i}}$ moves to larger $K$ values for band A, which means that the angular momentum begins to deviate from the s-l plane, i.e. the aplanar rotation takes place. For band B, the maximum probability for $K_{1}$ also move to larger $K$ values, which suggests the deviation of the angular momentum from the $\mathrm{s}-\mathrm{i}$ plane. Therefore, it is shown that with the increase of spin, aplanar rotation develops in both bands. The chiral symmetry breaks spontaneously, which is recognized as static chirality. The small finite probabilities for $K_{\mathrm{i}} \sim 0$ and $K_{1} \sim 0$ reflect the tunneling between the left- and right-handed configurations, which correspond to the energy separation between the near degenerating doublets.

For $I>14 \hbar$, the most probable value for $K_{\mathrm{i}}$ increase with spin for band A, while those for $K_{\mathrm{s}}$ and $K_{\mathrm{l}}$ almost stay constant. This suggests that the total angular momentum moves towards the i-axis as spin increases, by which the static chirality begins to disappear, while the tendency of principle axis rotation develops. Similar trend also exists for band B, which is not as significant as for band A.

\section{Azimuthal-Plot}

The chiral geometry in the intrinsic frame can also be illustrated by the so-called Azimuthal-Plot, which gives the distributions of the tilted angles $(\theta, \phi)$ of the angular momentum. The tilted angles $(\theta, \phi)$ are the polar angle and the azimuthal angle of the angular momentum in the intrinsic frame, respectively. As shown in Ref. [51], the tilted angles can be connected to the Euler angles $\left\{\psi^{\prime}, \theta^{\prime}, \phi^{\prime}\right\}$

$$
\theta=\theta^{\prime}, \quad \phi=\pi-\phi^{\prime},
$$

if the $z$-axis of the laboratory frame is chosen parallel to the angular momentum. Therefore, the distribution of the tilted angles can be obtained from those of the Euler angles. The details have been given in Ref. [51].

The calculated Azimuthal-Plots are shown for $I=10,12,14,16,18 \hbar$ in Fig. 2, from which one finds similar evolution of the angular momentum geometry to the $K$-Plots discussed above. For $I=10 \hbar$, the maximum probability appears at $\phi=90^{\circ}$ for band A, which corresponds to the s-l plane. The maximum probability for band $\mathrm{B}$ appears at $\theta=90^{\circ}$, corresponding to the $\mathrm{s}-\mathrm{i}$ plane. Therefore, the planar natural of rotation is confirmed by the Azimuthal-Plots at $I=10 \hbar$. 


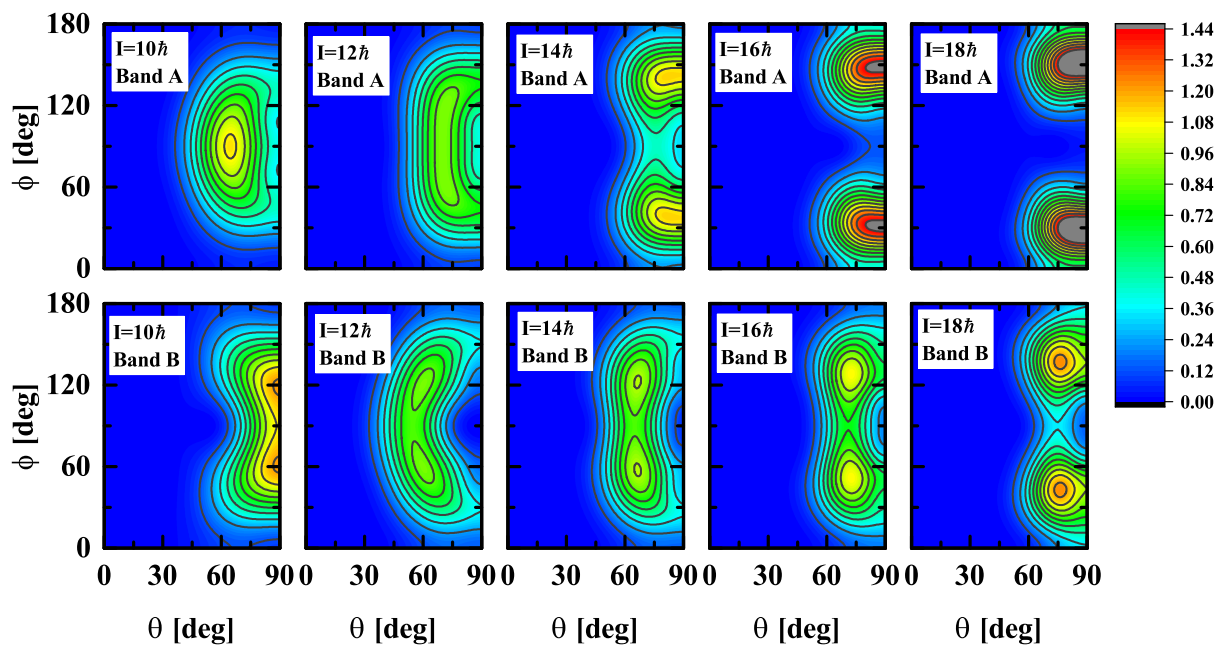

Fig. 2. (Color online) Azimuthal-Plots calculated at $I=10,12,14,16,18 \hbar$.

For $I=12,14 \hbar$, the peak found for band A at $I=10 \hbar$ splits into two, with their positions deviate from $\phi=90^{\circ}$, corresponding to the onset of aplanar rotation. The peaks for band $\mathrm{B}$ also move from $\theta=90^{\circ}$ to smaller $\theta$ values, which corresponds to aplanar orientations. The existence of the aplanar rotation in both bands demonstrates the occurrence of static chirality. The tunneling between the left- and right-handed configurations is reflected by the non-vanishing probabilities for either $\left(\theta=90^{\circ}, \phi=0^{\circ}\right)$ and $\left(\theta=90^{\circ}\right.$, $\left.\phi=180^{\circ}\right)$.

For higher spins, the peaks for band A move towards $\left(\theta=90^{\circ}, \phi=90^{\circ}\right)$, which corresponds to the i-axis, and the distribution becomes more and more concentrated. This corresponds to the tendency of principle axis rotation mentioned in the discussion of the $K$-Plots. The tendency is less significant for band $\mathrm{B}$ in which aplanar rotation remains until $I=18 \hbar$. However, the peaks for band $\mathrm{B}$ also get close to $\theta=90^{\circ}$ which corresponds to the $\mathrm{s}-\mathrm{i}$ plane, and its chirality is expected to disappear at even higher spins.

\section{Summary and outlook}

In this contribution, the angular momentum geometry in the intrinsic frame is extracted from the angular momentum projected wave functions in the laboratory frame with the $K$-Plots and the Azimuthal-Plots, which give the distributions of the components and the tilted angles of the angular momentum with respect to the three principle axes, respectively. The $K$-Plots and the Azimuthal-Plots are shown for the chiral doublet bands in ${ }^{128} \mathrm{Cs}$, which are described by the angular momentum projection approach 
based on the pairing-plus-quadrupole Hamiltonian. The results demonstrate the occurrence of chiral symmetry breaking for intermediate spins, in accordance with the established picture for the chiral doublet bands. It should be noted that the angular momentum projection approach used here could be combined with state-of-art mean-field descriptions, such as the energy density functionals, which enable the systematic description to chiral bands in various mass regions. The formalism for the $K$-Plots and the AzimuthalPlots can also be applied straightforwardly to other exotic rotating modes in triaxial nuclei, such as the wobbling mode, to examine the corresponding angular momentum geometries. Finally, the present study sets an example for the model in the laboratory frame to find their connections to the intuitive pictures proposed in the intrinsic frame.

We thank Q.B. Chen, Z.C. Gao, Y.A. Luo, F. Pan, Y. Sun, S.Y. Wang, and S.Q. Zhang for discussion and/or collaboration. This work was partly supported by the Chinese Major State 973 Program No. 2013CB834400, the National Natural Science Foundation of China (grants Nos. 11335002, 11375015, 11461141002, and 11621131001), and the China Postdoctoral Science Foundation under grants Nos. 2015M580007, 2016T90007 and 2017M610688.

\section{REFERENCES}

[1] S. Frauendorf, J. Meng, Nucl. Phys. A 617, 131 (1997).

[2] K. Starosta et al., Phys. Rev. Lett. 86, 971 (2001).

[3] S.Y. Wang et al., Phys. Lett. B 703, 40 (2011).

[4] C. Liu et al., Phys. Rev. Lett. 116, 112501 (2016).

[5] C. Vaman et al., Phys. Rev. Lett. 92, 032501 (2004).

[6] P. Joshi et al., Phys. Rev. Lett. 98, 102501 (2007).

[7] D. Tonev et al., Phys. Rev. Lett. 112, 052501 (2014).

[8] E.O. Lieder et al., Phys. Rev. Lett. 112, 202502 (2014).

[9] N. Rather et al., Phys. Rev. Lett. 112, 202503 (2014).

[10] I. Kuti et al., Phys. Rev. Lett. 113, 032501 (2014).

[11] S. Zhu et al., Phys. Rev. Lett. 91, 132501 (2003).

[12] D. Tonev et al., Phys. Rev. Lett. 96, 052501 (2006).

[13] C.M. Petrache, G.B. Hagemann, I. Hamamoto, K. Starosta, Phys. Rev. Lett. 96, 112502 (2006).

[14] E. Grodner et al., Phys. Rev. Lett. 97, 172501 (2006).

[15] S. Mukhopadhyay et al., Phys. Rev. Lett. 99, 172501 (2007). 
[16] A.D. Ayangeakaa et al., Phys. Rev. Lett. 110, 172504 (2013).

[17] D.L. Balabanski et al., Phys. Rev. C 70, 044305 (2004).

[18] E.A. Lawrie et al., Phys. Rev. C 78, 021305 (2008).

[19] J. Meng, S.Q. Zhang, J. Phys. G: Nucl. Part. Phys. 37, 064025 (2010).

[20] J. Meng, Q.B. Chen, S.Q. Zhang, Int. J. Mod. Phys. E 23, 1430016 (2014).

[21] J. Meng, P.W. Zhao, Phys. Scr. 91, 053008 (2016).

[22] S.Y. Wang, S.Q. Zhang, B. Qi, J. Meng, Phys. Rev. C 75, 024309 (2007).

[23] S.Q. Zhang, B. Qi, S.Y. Wang, J. Meng, Phys. Rev. C 75, 044307 (2007).

[24] S.Y. Wang et al., Phys. Rev. C 77, 034314 (2008).

[25] E.A. Lawrie, O. Shirinda, Phys Lett. B 689, 66 (2010).

[26] O. Shirinda, E.A. Lawrie, Eur. Phys. J. A 48, 118 (2012).

[27] B. Qi et al., Phys. Lett. B 675, 175 (2009).

[28] B. Qi et al., Phys. Rev. C 83, 034303 (2011).

[29] B. Qi et al., Phys. Rev. C 88, 027302 (2013).

[30] Q.B. Chen, J.M. Yao, S.Q. Zhang, B. Qi, Phys. Rev. C 82, 067302 (2010).

[31] I. Hamamoto, Phys. Rev. C 88, 024327 (2013).

[32] V.I. Dimitrov, S. Frauendorf, F. Dönau, Phys. Rev. Lett. 84, 5732 (2000).

[33] P. Olbratowski, J. Dobaczewski, J. Dudek, W. Płóciennik, Phys. Rev. Lett. 93, 052501 (2004).

[34] P. Olbratowski, J. Dobaczewski, J. Dudek, Phys. Rev. C 73, 054308 (2006).

[35] P.W. Zhao, Phys. Lett. B 773, 1 (2017).

[36] D. Almehed, F. Dönau, S Frauendorf, Phys. Rev. C 83, 054308 (2011).

[37] Q.B. Chen et al., Phys. Rev. C 87, 024314 (2013).

[38] Q.B. Chen et al., Phys. Rev. C 94, 044301 (2016).

[39] P. Ring, P. Schuck, The Nuclear Many-Body Problem, Springer, 1980.

[40] K. Hara, Y. Sun, Int. J. Mod. Phys. E 04, 637 (1995).

[41] B. Sabbey, M. Bender, G.F. Bertsch, P.-H. Heenen, Phys. Rev. C 75, 044305 (2007).

[42] T.R. Rodríguez, J. Luis Egido, Phys. Rev. C 81, 064323 (2010).

[43] T.R. Rodríguez, A. Arzhanov, G. Martínez-Pinedo, Phys. Rev. C 91, 044315 (2015).

[44] T. Nikšić, D. Vretenar, G.A. Lalazissis, P. Ring, Phys. Rev. Lett. 99, 092502 (2007).

[45] J.M. Yao, J. Meng, P. Ring, D. Vretenar, Phys. Rev. C 81, 044311 (2010).

[46] Y. Sun, Phys. Scr. 91, 043005 (2016).

[47] Z.-Ch. Gao, Y.S. Chen, Y. Sun, Phys. Lett. B 634, 195 (2006).

[48] J.A. Sheikh et al., Phys. Rev. C 77, 034313 (2008).

[49] G.H. Bhat, J.A. Sheikh, R. Palit, Phys. Lett. B 707, 250 (2012).

[50] G.H. Bhat, R.N. Ali, J.A. Sheikh, R. Palit, Nucl. Phys. A 922, 150 (2014).

[51] F.Q. Chen et al., Phys. Rev. C 96, 051303(R) (2017).

[52] T. Koike et al., Phys. Rev. C 67, 044319 (2003). 